QUARTERLY OF APPLIED MATHEMATICS

VOLUME LXIII, NUMBER 1

MARCH 2005, PAGES 71-87

S $0033-569 X(04) 00941-1$

Article electronically published on December 17, 2004

\title{
A STOCHASTIC CONTROL MODEL OF INVESTMENT, PRODUCTION AND CONSUMPTION
}

\author{
$\mathrm{BY}$ \\ WENDELL H. FLEMING (Division of Applied Mathematics, Brown University, Providence, RI \\ 02912) \\ AND
}

TAO PANG (Department of Mathematics, NC State University, Raleigh, NC 27695)

\begin{abstract}
We consider a stochastic control model in which an economic unit has productive capital and also liabilities in the form of debt. The worth of capital changes over time through investment as well as through random Brownian fluctuations in the unit price of capital. Income from production is also subject to random Brownian fluctuations. The goal is to choose investment and consumption controls which maximize total expected discounted HARA utility of consumption. Optimal control policies are found using the method of dynamic programming. In case of logarithmic utility, these policies have explicit forms.
\end{abstract}

1. Introduction. In this paper we consider a stochastic control model of the following kind. (Section 2 gives a detailed formulation of the model.) An economic unit has productive capital and also liabilities in the form of debt. The worth $K_{t}$ of capital at time $t$ changes through investment as well as through random fluctuations in the unit price of capital $P_{t}$. See equation (2.2). The debt $L_{t}$ owed by the economic unit changes through interest payments, investment, consumption, and income from production. Income from production $Y_{t}$ fluctuates randomly and it is proportional to the number of units of capital. See (2.3) and (2.4). The net worth of the economic unit is $X_{t}=K_{t}-L_{t}$. We require that $X_{t}>0$. The random fluctuations in the price $P_{t}$ and in the productivity of capital are modeled through Brownian motion inputs $\tilde{w}_{t}, w_{t}$ to the stochastic differential equations (2.7), (2.9) which $P_{t}$ and $X_{t}$ satisfy. The goal is to maximize the total discounted expected utility of consumption (2.10) subject to the constraint (2.11).

One interpretation of the model is as an extension of the classical Merton-type small investor portfolio optimization model. In this interpretation, $K_{t}$ is the investor's wealth in a risky asset and $-L_{t}$ is his wealth in a riskless asset. Income from production $Y_{t}$

Received April 22, 2004.

2000 Mathematics Subject Classification. Primary 60H30, 91B28, 93E20.

E-mail address: whf@cfm.brown.edu

E-mail address: tpang@math.ncsu.edu

(C)2004 Brown University 
represents dividends, which are proportional to the number of shares $N_{t}$ of the risky asset owned by the investor. Many modifications of the Merton models have been considered by several authors. In B-P, F-Sh2, continuous time portfolio optimization models of Merton type were considered, where the mean returns of individual asset categories are explicitly affected by underlying economic factors such as dividends and interest rates. The case of a Merton-type model with consumption and the interest rate which varies in a random, Markovian way, was considered in the authors' other papers [F-P, P1, P2]. In some other extension of the model, stochastic volatility is taken into consideration. See [F-HH, F-P-S Z].

In Section 3, the case of logarithmic utility function is considered. An elementary argument, using the Ito differential rule, gives an optimal investment control policy. This policy is also optimal for the problem of maximizing $E \log X_{T}$, the expected $\log$ utility of net worth at any time T. See Proposition 3.1. The optimal long term growth rate of $E \log X_{T}$ depends on the function $\mu(P)$ in the stochastic differential equation (2.7) for the unit capital price $P_{t}$. The optimal long term growth rate is found explicitly if $\mu(P)=\mu$ is a constant, or if $P_{t}$ is ergodic.

In Section 4, non-logarithmic HARA utility functions are considered, using the method of dynamic programming. The stochastic control problem has states $X_{t}, \lambda_{t}$ where $\lambda_{t}=$ $\log P_{t}$. The controls are $k_{t}, c_{t}$ where $C_{t}=c_{t} X_{t}$ is the consumption rate. For HARA utility, the dynamic programming equation for the value function $V(x, \lambda)$ reduces to the ordinary differential equation (4.9) for a function $W(\lambda)$, or equivalently, equation (4.11) for $Z(\lambda)=\log W(\lambda)$. By a sub/super solution method, a bounded solution $Z(\lambda)$ to (4.11) is found. Optimal investment and consumption control policies $k^{*}(\lambda)$ and $c^{*}(\lambda)$ are expressed in terms of $Z(\lambda)$ and its derivative $Z_{\lambda}(\lambda)$ via (4.31).

The work reported here was partly stimulated by a related study of macro-economic stochastic control models in international finance [F-St1, [F-St2]. In our model, and also in [F-St1, the ratio $k_{t}=K_{t} / X_{t}$ has the role of an investment control. The ratio $L_{t} / X_{t}$ of debt to net worth is $k_{t}-1$. Debt is often a useful means of economic development, leading to enhanced wealth and consumption. However, high levels of $L_{t} / X_{t}$ in fluctuating economic environments carry risks as well as benefits. We believe that there is a role for stochastic control models in addressing such issues. The model in this paper and in [F-St1] should be considered prototypes, which we hope will stimulate further work. See also comments in Section 5 in this regard.

2. Problem formulation. Let $N_{t}$ denote the number of the units of capital at time $t$ and $P_{t}$ the unit price of capital. Then the total worth of capital is

$$
K_{t}=N_{t} P_{t}
$$

Let $I_{t}$ denote the investment rate. Then

$$
d K_{t}=K_{t} \cdot \frac{d P_{t}}{P_{t}}+P_{t} d N_{t}=K_{t} \cdot \frac{d P_{t}}{P_{t}}+I_{t} d t .
$$


Let $Y_{t}$ denote the rate of income from production. We assume that $Y_{t}$ is proportional to the number of units $N_{t}$ of capital:

$$
Y_{t} d t=b_{t} N_{t} d t
$$

where $b_{t}$ is the productivity of capital. Let $L_{t}$ denote the debt and $C_{t}$ the consumption rate at time $t$. The balance equation for the change in debt is

$$
d L_{t}=\left[r_{t} L_{t}+I_{t}+C_{t}-Y_{t}\right] d t,
$$

where $r_{t}$ is the interest rate. In this paper, we assume that $r_{t}=r$ is a constant. However, both the unit price of capital $P_{t}$ and the productivity $b_{t}$ are random, as we shall specify.

Let $X_{t}$ denote the net worth of the economic unit at time t:

$$
X_{t}=K_{t}-L_{t} .
$$

We impose the constraints $C_{t} \geq 0, K_{t} \geq 0$ and $X_{t}>0$ ( or, equivalently, $L_{t}<K_{t}$ ). Note that $L_{t}$ can be positive or negative. If $L_{t}<0$, then $-L_{t}$ is the amount invested in a 'safe' financial asset, which earns interest at rate $r$. No bounds are imposed on the investment rate $I_{t}$. Let $k_{t}=K_{t} / X_{t}, c_{t}=C_{t} / X_{t}$. By subtracting (2.4) from (2.2), we obtain, with $r_{t}=r$

$$
d X_{t}=X_{t}\left[k_{t} \frac{d P_{t}}{P_{t}}-\left(r\left(k_{t}-1\right)+c_{t}-b_{t} \frac{k_{t}}{P_{t}}\right) d t\right] .
$$

We assume that $P_{t}$ satisfies a stochastic differential equation of the form

$$
d P_{t}=P_{t}\left[\mu\left(P_{t}\right) d t+\tilde{\sigma} d \tilde{w}_{t}\right],
$$

where $\tilde{\sigma}>0, \tilde{w}_{t}$ is a standard Brownian motion and $\mu(P)$ is such that (2.7) has a pathwise unique positive solution for any initial data $P_{0}>0$. If $\mu(P)=\mu$ is constant, then $P_{t}$ is a $\log$ Brownian motion. The productivity $b_{t}$ is subject to white-noise type fluctuations about a constant mean productivity $b>0$. More precisely, we assume that

$$
b_{t} d t=b d t+\sigma d w_{t},
$$

where $\sigma>0$ and $w_{t}$ is another standard Brownian motion. The Brownian motions $\tilde{w}_{t}, w_{t}$ may be correlated:

$$
E\left[\tilde{w}_{t} \cdot w_{t}\right]=\rho t, \quad|\rho| \leq 1 .
$$

The equation (2.6) now takes the precise form:

$$
\begin{aligned}
d X_{t}=X_{t} & {\left[\left(\mu\left(P_{t}\right)+\frac{b}{P_{t}}-r\right) k_{t} d t+\left(r-c_{t}\right) d t\right.} \\
& \left.+\tilde{\sigma} k_{t} d \tilde{w}_{t}+\frac{\sigma k_{t}}{P_{t}} d w_{t}\right] .
\end{aligned}
$$

The optimal stochastic control problem which we consider is the following. The controls are $k_{t}$ and $c_{t}$, which we call respectively investment and consumption controls. The state variables are $X_{t}, P_{t}$. The state dynamics are (2.7), (2.9). Let $U(C)$ denote a utility function. Then the goal is to choose controls to maximize total expected discounted utility of consumption:

$$
J=E \int_{0}^{\infty} e^{-\delta t} U\left(c_{t} X_{t}\right) d t, \quad \delta>0
$$


subject to the constraints

$$
c_{t} \geq 0, \quad k_{t} \geq 0, \quad X_{t}>0 .
$$

We require that the control $\left(k_{t}, c_{t} ; t \geq 0\right)$ is a $\mathbf{R}^{2}$-valued process. In addition, we require that $\left(k_{t}, c_{t}\right)$ is $\mathcal{F}_{t}$-progressively measurable for some $\left(\tilde{w}_{t}, w_{t}\right)$-adapted increasing family of $\sigma$-algebras $\left(\mathcal{F}_{t}, t \geq 0\right)$. See [F-So] Chapter 4 for details. In certain cases, $\left(k_{t}, c_{t}\right)$ can be obtained from a locally Lipschitz continuous control policy $(\underline{k}, \underline{c})$ :

$$
k_{t}=\underline{k}\left(t, X_{t}, P_{t}\right), \quad c_{t}=\underline{c}\left(t, X_{t}, P_{t}\right),
$$

where $X_{t}, P_{t}$ are obtained by substituting these policies in (2.7), (2.9).

We note that if productivity of capital is omitted from the model $(b=\sigma=0)$, then it reduces to the classical small-investor portfolio optimization problem, with the no shortselling constraint $k_{t} \geq 0$. For $\mu(P)=\mu$, a constant larger than the interest rate $r$, and for HARA utility $U(C)$, this problem has the familiar explicit Merton solution. When productivity of capital is included in the model, there is again an explicit solution for utility $U(C)=\log C$. See formula (3.7) below. However, for non-log HARA utility, this is no longer true (Section 4).

3. Log utility function. In this section, we consider the log utility function case

$$
U(C)=\log C .
$$

Moreover, we assume in this section that the ratio $c_{t}=C_{t} / X_{t}$ of consumption to net worth is a constant $c$. It will be seen that the optimal consumption control for the optimal stochastic control problem in Section 2 is $c_{t}^{*}=\delta$, when utility is logarithmic. See Remark 4.1 at the end of Section 4.

When $c_{t}=c$ is constant and utility is logarithmic, the optimal investment control policy can be found by the following elementary calculation. In (2.10),

$$
J=\frac{1}{\delta} \log c+\int_{0}^{\infty} e^{-\delta T} E \log X_{T} d T .
$$

By applying the Ito differential rule to $d \log X_{t}$ and using (2.9), we obtain

$$
E\left[\log X_{T}\right]=\log x_{0}+\int_{0}^{T} E\left[Q\left(k_{t}, P_{t}\right)\right] d t+(r-c) T,
$$

where $x_{0}=X_{0}$ is the initial net worth and

$$
\begin{aligned}
Q(k, P) & =-\frac{\alpha_{1}(P)}{2} k^{2}+\alpha_{2}(P) k, \\
\alpha_{1}(P) & =\frac{1}{P^{2}}\left(\tilde{\sigma}^{2} P^{2}+2 \rho \sigma \tilde{\sigma} P+\sigma^{2}\right), \\
\alpha_{2}(P) & =\mu(P)+\frac{b}{P}-r .
\end{aligned}
$$

In this paper, we consider $-1<\rho \leq 1$. From (2.7), we can show that $P_{t}>0$ as long as $P_{0}>0$, which is a reasonable assumption. Therefore, it is not hard to verify that 
$\alpha_{1}(P)>0$, provided that $P>0,-1<\rho \leq 1$. Thus, for each $P, Q(k, P)$ is maximized subject to the constraint $k \geq 0$, when $k=k^{*}(P)$, where

$$
k^{*}(P)= \begin{cases}\frac{\alpha_{2}(P)}{\alpha_{1}(P)}, & \text { if } \alpha_{2}(P) \geq 0 \\ 0, & \text { if } \alpha_{2}(P)<0\end{cases}
$$

By $(3.3), E\left[\log X_{t}\right]$ is maximized by taking $k_{t}=k^{*}\left(P_{t}\right)$ for $0 \leq t \leq T$. Therefore, we have

Proposition 3.1. For any $T>0$ and constant consumption control $\left(c_{t}=c\right.$ for $0 \leq t \leq$ $T)$, the investment control $k^{*}=k^{*}\left(P_{t}\right)$ for $0 \leq t \leq T$ maximizes $E\left[\log X_{T}\right]$.

Note that the optimal control policy $k^{*}(\cdot)$ is stationary (not time-dependent). Moreover, $k^{*}(\cdot)$ does not depend on $c$. From (3.2), we also have:

Corollary 3.2. For each constant consumption control $c$, the investment control $k_{t}^{*}=$ $k^{*}\left(P_{t}\right)$ for all $t \geq 0$ maximizes

$$
J=E \int_{0}^{\infty} e^{-\delta t} \log \left(c_{t} X_{t}\right) d t .
$$

Let us return to the stochastic control problem in Section 2, in which the consumption control $c_{t}$ need not be constant. As already mentioned, the method of dynamic programming gives the (well-known) result that the optimal consumption control is $c_{t}^{*}=\delta$, which is constant. Thus, for log utility, the optimal controls for this problem are

$$
k_{t}^{*}=k^{*}\left(P_{t}\right), \quad c_{t}^{*}=\delta
$$

where $k^{*}(P)$ is given by (3.7). See Remark 4.1 at the end of Section 4 .

Optimal long term growth of $E\left[\log X_{t}\right]$. Let us again choose $c_{t}=c$ to be any constant consumption control and choose $k_{t}=k_{t}^{*}=k^{*}\left(P_{t}\right)$ optimally as in Proposition 3.1. By $(3.3)$,

$$
\begin{aligned}
E\left[\log X_{T}\right] & =\log x_{0}+\int_{0}^{T} E\left[\Phi\left(P_{t}\right)\right] d t+(r-c) T \\
\Phi(P) & =Q\left(k^{*}(P), P\right) .
\end{aligned}
$$

The growth rate as $T \rightarrow \infty$ of $E\left[\log X_{T}\right]$ depends on the behavior of $E\left[\Phi\left(P_{t}\right)\right]$ for large $t$. We analyze this only in two special cases.

Case 1. $\mu(P)=\mu$ is constant and $\mu>r$. By (2.7), $P_{t}$ is then a log Brownian motion. Moreover,

$$
\begin{aligned}
k^{*}(P) & =\frac{(b+(\mu-r) P) P}{\sigma^{2}+2 \rho \sigma \tilde{\sigma} P+\tilde{\sigma}^{2} P^{2}} \\
\Phi(P) & =\frac{(b+(\mu-r) P)^{2}}{2\left(\sigma^{2}+2 \rho \sigma \tilde{\sigma} P+\tilde{\sigma}^{2} P^{2}\right)} .
\end{aligned}
$$


The function $\Phi(P)$ is bounded and has limit $(\mu-r)^{2} /\left(2 \tilde{\sigma}^{2}\right)$ as $P \rightarrow \infty$. Since $\mu>r, P_{t}$ tends to infinity almost surely as $t \rightarrow \infty$. Hence

$$
\begin{aligned}
\lim _{t \rightarrow \infty} E\left[\Phi\left(P_{t}\right)\right] & =\frac{(\mu-r)^{2}}{2 \tilde{\sigma}^{2}}, \\
\lim _{T \rightarrow \infty} \frac{1}{T} E\left[\log X_{T}\right] & =\frac{(\mu-r)^{2}}{2 \tilde{\sigma}^{2}}+r-c .
\end{aligned}
$$

This is the same result as for the classical Merton model, with $b=\sigma=0$. Note also that $k^{*}(P)$ tends to $(\mu-r) /\left(\tilde{\sigma}^{2}\right)$ as $P \rightarrow \infty$. This limit is the classical Merton result for $\log$ utility.

Case 2. $P_{t}$ is ergodic. In this case

$$
\lim _{T \rightarrow \infty} \frac{1}{T} E\left[\log X_{T}\right]=E_{e q} \Phi+r-c .
$$

Example 3.3. Let

$$
\mu(P)=-a \log \frac{P}{\bar{P}}+\frac{1}{2} \tilde{\sigma}^{2},
$$

where $a, \bar{P}$ are positive constants. Then $\lambda_{t}=\log P_{t}$ satisfies the linear stochastic differential equation

$$
d \lambda_{t}=-a\left(\lambda_{t}-\bar{\lambda}\right) d t+\tilde{\sigma} d \tilde{w}_{t},
$$

where $\bar{\lambda}=\log \bar{P}$. The equilibrium distribution of $\lambda_{t}$ is Gaussian with mean $\bar{\lambda}$ and variance $\tilde{\sigma}^{2} /(2 a)$, from which the equilibrium distribution of $P_{t}$ can be calculated. This model for $P_{t}$ is of the type considered by Platen-Rebolledo [P-R] and also Fleming-Sheu [F-Sh1, after a constant linear trend in $\lambda_{t}$ has been removed.

4. Non-log HARA utility function. In this section, we consider the non-log HARA utility case:

$$
U(C)=\frac{1}{\gamma} C^{\gamma}, \quad-\infty<\gamma<1, \gamma \neq 0
$$

For simplicity, let us again take $r_{t} \equiv r$, which is a constant. We also assume that $\mu(P)=\mu$ is a constant and $\mu>r$ in this section.

Assume that the equations of $K_{t}, L_{t}, P_{t}, b_{t}, X_{t}$ are still given by (2.2), (2.4), (2.7), (2.8) and (2.5). The goal is to maximize the objective function

$$
J=E \int_{0}^{\infty} e^{-\delta t} \frac{1}{\gamma} C_{t}^{\gamma} d t
$$

subject to the constraints $K_{t} \geq 0, C_{t} \geq 0, X_{t}>0$.

As in Section 2, we take $c_{t} \equiv \frac{C_{t}}{X_{t}}, k_{t} \equiv \frac{K_{t}}{X_{t}}$ as our controls. Then the equation of $X_{t}$ can be written as

$$
d X_{t}=X_{t}\left[\left(\mu+\frac{b}{P_{t}}-r\right) k_{t} d t+\left(r-c_{t}\right) d t+k_{t}\left(\tilde{\sigma} d \tilde{w}_{t}+\frac{\sigma}{P_{t}} d w_{t}\right)\right]
$$

where $\tilde{w}_{t}$ and $w_{t}$ are two standard Brownian motions that are correlated with each other:

$$
E\left[d \tilde{w}_{t} \cdot d w_{t}\right]=\rho d t, \quad-1<\rho \leq 1 .
$$

Let

$$
\lambda_{t}=\log P_{t}, \quad y_{t}=e^{-\lambda_{t}}=P_{t}^{-1}
$$


Then, by virtue of (2.7), we can get

$$
d \lambda_{t}=\tilde{\mu} d t+\tilde{\sigma} d \tilde{w}_{t}
$$

where $\tilde{\mu}=\mu-\frac{1}{2} \tilde{\sigma}^{2}$. The equation of $X_{t}$ can be rewritten as

$$
d X_{t}=X_{t}\left[\left(\mu+b y_{t}-r\right) k_{t} d t+\left(r-c_{t}\right) d t+k_{t}\left(\tilde{\sigma} d \tilde{w}_{t}+\sigma y_{t} d w_{t}\right)\right] .
$$

Use $X_{t}, \lambda_{t}$ as the state variables. The value function is

$$
V(x, \lambda)=\sup _{c_{t} \geq 0, k_{t} \geq 0} E_{x, \lambda} \int_{0}^{\infty} e^{-\delta t} \frac{1}{\gamma}\left(c_{t} X_{t}\right)^{\gamma} d t
$$

where $x, \lambda$ are the initial values of $X_{t}, \lambda_{t}$. For non-log HARA utility function, it is not hard to show that $V(x, \lambda)$ is homogeneous in $x$ with an order of $\gamma$. Therefore the value function has the form

$$
V(x, \lambda)=\frac{W(\lambda)}{\gamma} x^{\gamma}
$$

By virtue of Ito's rule, we can write the dynamic programming equation for $V(x, \lambda)$ as

$$
\begin{aligned}
& \delta V=\frac{\tilde{\sigma}^{2}}{2} V_{\lambda \lambda}+\tilde{\mu} V_{\lambda}+r x V_{x}+\max _{c \geq 0}\left[-c x V_{x}+\frac{1}{\gamma}(c x)^{\gamma}\right] \\
& +\max _{k \geq 0}\left[(b y+\mu-r) k x V_{x}+\left(\tilde{\sigma}^{2}+\rho \sigma \tilde{\sigma} y\right) k x V_{x \lambda}+\frac{k^{2} x^{2} q(y)}{2} V_{x x}\right],
\end{aligned}
$$

where

$$
q(y)=\sigma^{2} y^{2}+2 \rho \sigma \tilde{\sigma} y+\tilde{\sigma}^{2} .
$$

We assume that $\rho \neq-1$, so that $q(y)>0$. From (4.6), we can get that the reduced DPE for $W$ is

$$
\begin{aligned}
\delta W= & \gamma \max _{k \geq 0}\left[(b y+\mu-r) k W+\left(\tilde{\sigma}^{2}+\rho \sigma \tilde{\sigma} y\right) k W_{\lambda}-\frac{k^{2}}{2}(1-\gamma) q(y) W\right] \\
& +\frac{\tilde{\sigma}^{2}}{2} W_{\lambda \lambda}+\tilde{\mu} W_{\lambda}+\gamma r W+(1-\gamma) W^{\frac{\gamma}{\gamma-1}} .
\end{aligned}
$$

Let $Z=\log W$. Then we have

So the equation of $Z$ is

$$
\frac{W_{\lambda}}{W}=Z_{\lambda}, \quad \frac{W_{\lambda \lambda}}{W}=Z_{\lambda \lambda}+Z_{\lambda}^{2}
$$

$$
\begin{aligned}
\delta= & \frac{\tilde{\sigma}^{2}}{2}\left(Z_{\lambda \lambda}+Z_{\lambda}^{2}\right)+\tilde{\mu} Z_{\lambda}+\gamma r+(1-\gamma) e^{\frac{Z}{\gamma-1}} \\
& +\gamma \max _{k \geq 0}\left\{\left[b y+\mu-r+\left(\tilde{\sigma}^{2}+\rho \sigma \tilde{\sigma} y\right) Z_{\lambda}\right] k-\frac{k^{2}}{2}(1-\gamma) q(y)\right\} .
\end{aligned}
$$

The maximum occurs at

$$
k^{*}(\lambda)=\left[\frac{b y+\mu-r+\left(\tilde{\sigma}^{2}+\rho \sigma \tilde{\sigma} y\right) Z_{\lambda}}{(1-\gamma) q(y)}\right]^{+},
$$

where $a^{+} \equiv \max (a, 0)$. Define

$$
G(\lambda, p)=\max _{k \geq 0}\left\{\left[b y+\mu-r+\left(\tilde{\sigma}^{2}+\rho \sigma \tilde{\sigma} y\right) p\right] k-\frac{k^{2}}{2}(1-\gamma) q(y)\right\} .
$$


Then the equation (4.11) can be rewritten as

$$
\delta=\frac{\tilde{\sigma}^{2}}{2}\left(Z_{\lambda \lambda}+Z_{\lambda}^{2}\right)+\tilde{\mu} Z_{\lambda}+\gamma r+(1-\gamma) e^{\frac{Z}{\gamma-1}}+\gamma G\left(\lambda, Z_{\lambda}\right) .
$$

In addition, denote $\Psi$ as follows:

$$
\Psi(\lambda)=\frac{(b y+\mu-r)^{2}}{2 q(y)(1-\gamma)} .
$$

Then it is easy to verify that

$$
G(\lambda, p) \geq 0, \quad G(\lambda, 0)=\Psi(\lambda) .
$$

Define

$$
H(\lambda, z, p) \equiv-\frac{\tilde{\sigma}^{2}}{2} p^{2}-\tilde{\mu} p-\gamma G(\lambda, p)-\gamma r+\delta-(1-\gamma) e^{\frac{z}{\gamma-1}} .
$$

It is easy to show that $H$ is a continuous function. In addition, equation (4.14) can be written as

$$
\frac{\tilde{\sigma}^{2}}{2} Z_{\lambda \lambda}=H\left(\lambda, Z, Z_{\lambda}\right)
$$

DEFINITION 4.1. $Z(\lambda)$ is a subsolution (supersolution) of (4.18), provided that

$$
\frac{\tilde{\sigma}^{2}}{2} Z_{\lambda \lambda} \geq(\leq) H\left(\lambda, Z, Z_{\lambda}\right) .
$$

Next, we will show that (4.18) possesses a classical solution, by virtue of the sub/super solution method. For details about sub/super solution method, please refer to Fleming and Pang [F-P] Section 3.

4.1. $\gamma>0$ case. We consider the case that $0<\gamma<1$. We need to get a pair of ordered subsolution and supersolution to obtain the existence results of equation (4.18).

First let us get some information about $\Psi$. Actually, since $\mu$ is a constant, it is not hard to verify that $\Psi$ is bounded. More particularly, if $0 \leq \rho \leq 1$, since $y=e^{-\lambda}>0$, we have

$$
\begin{aligned}
\Psi(\lambda) & =\frac{(b y+\mu-r)^{2}}{2(1-\gamma)\left(\sigma^{2} y^{2}+2 \rho \sigma \tilde{\sigma} y+\tilde{\sigma}^{2}\right)} \\
& \leq \frac{(b y+\mu-r)^{2}}{2(1-\gamma)\left(\sigma^{2} y^{2}+\tilde{\sigma}^{2}\right)} \\
& \leq \frac{2 b^{2} y^{2}+2(\mu-r)^{2}}{2(1-\gamma)\left(\sigma^{2} y^{2}+\tilde{\sigma}^{2}\right)} \\
& =\frac{b^{2}}{\sigma^{2}(1-\gamma)}+\frac{(\mu-r)^{2}-\frac{b^{2} \tilde{\sigma}^{2}}{\sigma^{2}}}{(1-\gamma)\left(\sigma^{2} y^{2}+\tilde{\sigma}^{2}\right)}
\end{aligned}
$$

If $(\mu-r)^{2}-b^{2} \tilde{\sigma}^{2} / \sigma^{2} \leq 0$, then we have

$$
\Psi(\lambda) \leq \frac{b^{2}}{\sigma^{2}(1-\gamma)}
$$

Otherwise, we will have

$$
\Psi(\lambda) \leq \frac{b^{2}}{\sigma^{2}(1-\gamma)}+\frac{(\mu-r)^{2}-\frac{b^{2} \tilde{\sigma}^{2}}{\sigma^{2}}}{(1-\gamma) \tilde{\sigma}^{2}}=\frac{(\mu-r)^{2}}{\tilde{\sigma}^{2}(1-\gamma)} .
$$


That is, in case $0 \leq \rho \leq 1$, we can get

$$
\Psi(\lambda) \leq \max \left\{\frac{b^{2}}{\sigma^{2}(1-\gamma)}, \frac{(\mu-r)^{2}}{\tilde{\sigma}^{2}(1-\gamma)}\right\} .
$$

In addition, if $-1<\rho<0$, we have

$$
2 \rho \sigma \tilde{\sigma} y \geq \rho \sigma^{2} y^{2}+\rho \tilde{\sigma}^{2} .
$$

Therefore, for $-1<\rho<0$, we can get

$$
\begin{aligned}
\Psi(\lambda) & =\frac{(b y+\mu-r)^{2}}{2(1-\gamma)\left(\sigma^{2} y^{2}+2 \rho \sigma \tilde{\sigma} y+\tilde{\sigma}^{2}\right)} \\
& \leq \frac{(b y+\mu-r)^{2}}{2(1-\gamma)(1+\rho)\left(\sigma^{2} y^{2}+\tilde{\sigma}^{2}\right)} \\
& \leq \frac{2 b^{2} y^{2}+2(\mu-r)^{2}}{2(1-\gamma)(1+\rho)\left(\sigma^{2} y^{2}+\tilde{\sigma}^{2}\right)} \\
& \leq \frac{1}{1+\rho} \max \left\{\frac{b^{2}}{\sigma^{2}(1-\gamma)}, \frac{(\mu-r)^{2}}{\tilde{\sigma}^{2}(1-\gamma)}\right\} .
\end{aligned}
$$

Let

$$
\tilde{\Psi} \equiv \max \left\{\frac{b^{2}}{\sigma^{2}(1-\gamma)}, \frac{(\mu-r)^{2}}{\tilde{\sigma}^{2}(1-\gamma)}\right\}
$$

and

$$
\bar{\Psi} \equiv \max \left\{\tilde{\Psi}, \frac{\tilde{\Psi}}{1+\rho}\right\} \text {. }
$$

Then we can get that

$$
0 \leq \Psi(\lambda) \leq \bar{\Psi}<\infty .
$$

Lemma 4.1. Suppose $0<\gamma<1$ and

$$
\delta>\gamma r
$$

In addition, define

$$
K_{1} \equiv(\gamma-1) \log \left[\frac{\delta-\gamma r}{1-\gamma}\right]
$$

Then any constant $K \leq K_{1}$ is a subsolution of (4.18).

This is easily verified, so the proof is omitted here.

Lemma 4.2. Suppose $0<\gamma<1$ and

$$
\delta>\gamma(r+\bar{\Psi}),
$$

where $\bar{\Psi}$ is defined by (4.21). In addition, define

$$
K_{2} \equiv(\gamma-1) \log \left[\frac{\delta-\gamma(r+\bar{\Psi})}{1-\gamma}\right] .
$$

Then any constant $K \geq K_{2}$ is a supersolution of (4.18).

The proof is omitted here. 
Lemma 4.3. Suppose $0<\gamma<1$ and (4.25) holds. Then $K_{2} \geq K_{1}$, where $K_{1}, K_{2}$ are defined by (4.24) and (4.26) respectively. Moreover, (4.18) has a bounded classical solution $\tilde{Z}(\lambda)$ which satisfies

$$
K_{1} \leq \tilde{Z}(\lambda) \leq K_{2} .
$$

Proof. From (4.22), we can know that (4.25) implies (4.23). In addition, we can get $K_{2} \geq K_{1}$. Therefore, by virtue of Lemma 4.1 and Lemma $4.2,\left\langle K_{1}, K_{2}\right\rangle$ is a pair of ordered subsolution and supersolution of (4.18).

In addition, it is not hard to verify that

$$
\begin{aligned}
0 & \leq \max _{k \geq 0}\left\{\left[b y+\mu-r+\left(\tilde{\sigma}^{2}+\rho \sigma \tilde{\sigma} y\right) p\right] k-\frac{k^{2}}{2}(1-\gamma) q(y)\right\} \\
& \leq \frac{\left(b y+\mu-r+\left(\tilde{\sigma}^{2}+\rho \sigma \tilde{\sigma} y\right) p\right)^{2}}{2(1-\gamma) q(y)} \\
& \leq \frac{2(b y+\mu-r)^{2}+2\left(\tilde{\sigma}^{2}+\rho \sigma \tilde{\sigma} y\right)^{2} p^{2}}{2(1-\gamma) q(y)} \\
& =2 \Psi(\lambda)+\frac{2\left(\tilde{\sigma}^{2}+\rho \sigma \tilde{\sigma} y\right)^{2} p^{2}}{2(1-\gamma) q(y)}
\end{aligned}
$$

where $\Psi(\lambda)$ is given by (4.15). For $\rho \in(-1,1]$, it is not hard to show that $q(y)$ is bounded below by a positive constant. Moreover, there exists a positive constant $\tilde{C}_{1}$ such that

$$
\frac{2\left(\tilde{\sigma}^{2}+\rho \sigma \tilde{\sigma} y\right)^{2}}{2(1-\gamma) q(y)}<\tilde{C}_{1}
$$

Thus, by virtue of (4.22), we can get that there exists a positive constant $\tilde{C}_{2}$, such that

$$
0 \leq G(\lambda, p) \leq \tilde{C}_{1} p^{2}+\tilde{C}_{2} .
$$

Combined with (4.17) and (4.27), the above inequality implies

$$
|H(\lambda, z, p)| \leq C_{1}\left(p^{2}+C_{2}\right), \quad z \in\left[K_{1}, K_{2}\right],
$$

where $K_{1}, K_{2}$ are given by $(4.24),(4.26)$ and $C_{1}, C_{2}$ are two positive constants. Therefore, by virtue of Fleming and Pang [F-P] Section 3 Theorem 1, (4.18) has a classical solution $\tilde{Z}(\lambda)$ such that

$$
K_{1} \leq \tilde{Z}(\lambda) \leq K_{2} .
$$

Then we are done.

Definition 4.2 (Admissible Control Space). $\left(k_{t}, c_{t}\right)$ is said to be in the admissible control space $\Pi$ if $\left(k_{t}, c_{t}\right)$ is a $\mathbf{R}^{2}$-process which is progressively measurable with respect to a $\left(w_{t}, \tilde{w}_{t}\right)$-adapted family of $\sigma$-algebras $\left(\mathcal{F}_{t}, t \geq 0\right)$. Moreover, we require that

$$
\operatorname{Pr}\left(\int_{0}^{T} k_{t}^{2} d t<\infty\right)=1, \quad \operatorname{Pr}\left(\int_{0}^{T} c_{t} d t<\infty\right)=1,
$$

so that (4.2), (4.4) have solutions.

Lemma 4.4. Suppose $0<\gamma<1$ and (4.25) holds. If $\tilde{Z}(\lambda)$ is a classical solution of (4.18) which satisfies (4.27), then $\tilde{Z}_{\lambda}$ is bounded. 
Proof. From (4.27), we can see that $\tilde{Z}(\lambda)$ is bounded. Therefore, to show that $\tilde{Z}_{\lambda}(\lambda)$ is bounded, it is sufficient to show that $\tilde{Z}_{\lambda}(\lambda)$ is bounded at its local maxima and minima.

Suppose $\tilde{Z}_{\lambda}(\lambda)$ has a local maximum or minimum at $\lambda_{0}$. Then we have $\tilde{Z}_{\lambda \lambda}\left(\lambda_{0}\right)=0$. Therefore, at $\lambda=\lambda_{0}$, we can get

$$
\frac{\tilde{\sigma}^{2}}{2} \tilde{Z}_{\lambda}^{2}+\tilde{\mu} \tilde{Z}_{\lambda}+G\left(\lambda, \tilde{Z}_{\lambda}\right)+\gamma r-\delta+e^{\frac{\tilde{Z}}{\gamma-1}}=0,
$$

where $G(\lambda, p)$ and $\Psi(\lambda)$ are given by (4.13) and (4.15), respectively. Since $G(\lambda, p) \geq 0$, we have

$$
\frac{\tilde{\sigma}^{2}}{2} \tilde{Z}_{\lambda}^{2}+\tilde{\mu} \tilde{Z}_{\lambda}+\gamma r-\delta+e^{\frac{\tilde{z}}{\gamma-1}} \leq 0 .
$$

Moreover, by virtue of (4.22) and (4.27), we know that $\gamma r-\delta+e^{\frac{\tilde{Z}}{\gamma-1}}$ is also bounded. Therefore, from equation (4.28), it is not hard to show that $\tilde{Z}_{\lambda}$ is bounded at $\lambda=\lambda_{0}$. Since $\lambda_{0}$ is an arbitrary maximum or minimum point of $\tilde{Z}_{\lambda}, \tilde{Z}_{\lambda}$ is bounded at its maxima and minima. Thus, $\tilde{Z}_{\lambda}$ is bounded.

Theorem 4.1 (Verification Theorem). Suppose $\gamma>0$ and (4.25) holds. Let $\tilde{Z}(\lambda)$ denote a classical solution of (4.18) which satisfies (4.27). Denote

$$
\tilde{V}(x, \lambda) \equiv \frac{1}{\gamma} x^{\gamma} e^{\tilde{Z}(\lambda)}
$$

Then we have

$$
\tilde{V}(x, \lambda) \equiv V(x, \lambda)
$$

where $V(x, \lambda)$ is the value function defined by (4.6). Moreover, the optimal control policy is

$$
k^{*}(\lambda)=\left[\frac{b y+\mu-r+\left(\tilde{\sigma}^{2}+\rho \sigma \tilde{\sigma} y\right) \tilde{Z}_{\lambda}(\lambda)}{(1-\gamma) q(y)}\right]^{+}, \quad c^{*}(\lambda)=e^{\frac{\tilde{Z}(\lambda)}{\gamma-1}} .
$$

Proof. Since $\tilde{Z}$ is a classical solution of (4.18), by definition, it is easy to verify that $\tilde{V}(x, \lambda)$ is a classical solution of (4.7). For any admissible control $\left(k_{t}, c_{t}\right) \in \Pi$, by applying Ito's rule, we can get that

$$
d\left[e^{-\delta t} \tilde{V}\left(X_{t}, \lambda_{t}\right]=-\delta e^{-\delta t} \tilde{V}\left(X_{t}, \lambda_{t}\right) d t+e^{-\delta t} d \tilde{V}\left(X_{t}, \lambda_{t}\right) .\right.
$$

Since $\tilde{V}$ is a solution of (4.7), we can get

$$
\tilde{V}(x, \lambda) \geq \int_{0}^{T} e^{-\delta t} \frac{1}{\gamma}\left(c_{t} X_{t}\right)^{\gamma} d t+e^{-\delta T} \tilde{V}\left(X_{T}, \lambda_{T}\right)+m_{T}+\tilde{m}_{T},
$$

where $m_{T}, \tilde{m}_{T}$ are local martingales.

Let $\tau_{R}$ denote the exit time of $\left(X_{t}, \lambda_{t}\right)$ from $\left\{x^{2}+\lambda^{2} \leq R^{2}\right\}$. From (4.32), we can get

$$
\tilde{V}(x, \lambda) \geq E \int_{0}^{T \wedge \tau_{R}} e^{-\delta t} \frac{1}{\gamma}\left(c_{t} X_{t}\right)^{\gamma} d t+E e^{-\delta T \wedge \tau_{R}} \tilde{V}\left(X_{T \wedge \tau_{R}}, \lambda_{T \wedge \tau_{R}}\right) .
$$

Since $\tilde{V}>0$, let $R \rightarrow \infty$, and by virtue of Fatou's lemma, we can get

$$
\tilde{V}(x, \lambda) \geq E \int_{0}^{T} e^{-\delta t} \frac{1}{\gamma}\left(c_{t} X_{t}\right)^{\gamma} d t
$$


Let $T \rightarrow \infty$. By the definition of $V$, we can get

$$
\tilde{V}(x, \lambda) \geq V(x, \lambda) .
$$

On the other hand, for $\left(k^{*}, c^{*}\right)$ given by $(4.31)$, it is not hard to verify that $\left(k^{*}, c^{*}\right) \in \Pi$. In this case, (4.32) becomes an equality

$$
\tilde{V}(x, \lambda)=\int_{0}^{T} e^{-\delta t} \frac{1}{\gamma}\left(c_{t}^{*} X_{t}\right)^{\gamma} d t+e^{-\delta T} \tilde{V}\left(X_{T}, \lambda_{T}\right)+m_{T}+\tilde{m}_{T},
$$

where

$$
\begin{aligned}
& m_{T}=\int_{0}^{T} \sigma y_{t} k_{t}^{*} X_{t} \tilde{V}_{x}\left(X_{t}, \lambda_{t}\right) d w_{t}, \\
& \tilde{m}_{T}=\int_{0}^{T} \tilde{\sigma}\left(k_{t}^{*} X_{t} \tilde{V}_{x}\left(X_{t}, \lambda_{t}\right)+\tilde{V}_{\lambda}\left(X_{t}, \lambda_{t}\right)\right) d \tilde{w}_{t} .
\end{aligned}
$$

Since $\tilde{Z}, \tilde{Z}_{\lambda}$ are bounded, from (4.31), we can show that $k_{t}^{*}, y_{t} k_{t}^{*}$ and $c_{t}^{*}$ are all bounded. Therefore, from (4.4), under the control policy (4.31), it is not hard to show that

$$
E\left[X_{t}^{m}\right]<\infty, \quad \forall m>0 .
$$

Given this, by virtue of $(4.29),(4.36),(4.37)$, we can get that $m_{T}, \tilde{m}_{T}$ are martingales. Therefore, by taking expectation to (4.35), we have

$$
\tilde{V}(x, \lambda)=E \int_{0}^{T} e^{-\delta t} \frac{1}{\gamma}\left(c_{t}^{*} X_{t}\right)^{\gamma} d t+E e^{-\delta T} \tilde{V}\left(X_{T}, \lambda_{T}\right) .
$$

From (4.34), we can get that

$$
E \int_{0}^{\infty} e^{-\delta t} \frac{1}{\gamma}\left(c_{t}^{*} X_{t}\right)^{\gamma} d t<\infty
$$

Therefore,

$$
\liminf _{T \rightarrow \infty} E e^{-\delta T} \frac{1}{\gamma}\left(c_{T}^{*} X_{T}\right)^{\gamma}=0 .
$$

By virtue of (4.31) and (4.27), we have

$$
c_{t}^{*} \geq \underline{c}>0,
$$

where $\underline{c}$ is a constant. Thus, we can get

$$
\liminf _{T \rightarrow \infty} E e^{-\delta T} \frac{1}{\gamma} X_{T}^{\gamma}=0 .
$$

Combined with (4.27) and (4.29), this implies

$$
\liminf _{T \rightarrow \infty} E e^{-\delta T} \tilde{V}\left(X_{T}, \lambda_{T}\right)=0 .
$$

Taking liminf as $T \rightarrow \infty$ in (4.39), we can get

$$
\tilde{V}(x, \lambda)=E \int_{0}^{\infty} e^{-\delta t} \frac{1}{\gamma}\left(c_{t} X_{t}\right)^{\gamma} d t .
$$

By the definition of $V(X, \lambda)$, we have

$$
\tilde{V}(x, \lambda) \leq V(x, \lambda),
$$

which implies (4.30), combined with (4.34). 
4.2. $\gamma<0$ Case. In case of $\gamma<0$, it is not hard to verify that (4.22) still holds. Similar to the $\gamma>0$ case, we can also get a pair of ordered subsolution and supersolution. The results are as follows.

Lemma 4.5. Suppose $\gamma<0$. Define

$$
K_{1} \equiv(\gamma-1) \log \left[\frac{\delta-\gamma(r+\bar{\Psi})}{1-\gamma}\right]
$$

where $\bar{\Psi}$ are given by (4.20). Then any constant $K \leq K_{1}$ is a subsolution of (4.18).

Lemma 4.6. Suppose $\gamma<0$. Define

$$
K_{2} \equiv(\gamma-1) \log \left[\frac{\delta-\gamma r}{1-\gamma}\right]
$$

Then any constant $K \geq K_{2}$ is a supersolution of (4.18).

The proofs are rather standard, and they are omitted here.

Since $\gamma<0$, it is easy to verify that $K_{2}>K_{1}$. Therefore, $\left\langle K_{1}, K_{2}\right\rangle$ is a pair of sub and super solutions of (4.18). Then, similarly to Lemma 4.3, we have the following lemma.

Lemma 4.7. Suppose $\gamma<0$. Then (4.18) has a solution $\tilde{Z}$ which satisfies

$$
K_{1} \leq \tilde{Z}(\lambda) \leq K_{2}, \quad \forall \lambda,
$$

where $K_{1}, K_{2}$ are given by (4.41) and (4.42).

The proof is almost the same as the proof of Lemma 4.3. In addition, using the same techniques as in the proof of Lemma 4.5, we have

Lemma 4.8. Suppose $\tilde{Z}$ is a classical solution of (4.18) which satisfies (4.43). Then $\tilde{Z}_{\lambda}$ is bounded.

Before we go to the verification theorem, let us define the admissible control space first.

Definition 4.3. A progressive measurable control $\left(k_{t}, c_{t}\right)$ is said to be in the admissible control space $\Pi$ if $k_{t}, e^{-\lambda_{t}} k_{t}, c_{t}$ are bounded and

$$
c_{t} \geq \underline{c}>0 \text {. }
$$

The verification theorem is as follows.

TheOREM 4.2 (Verification Theorem). Suppose $\gamma<0$ and $\tilde{Z}$ is a classical solution of (4.18) which satisfies (4.43). Denote

$$
\tilde{V}(x, \lambda) \equiv \frac{1}{\gamma} x^{\gamma} e^{\tilde{Z}(\lambda)}
$$

Then we have

$$
\tilde{V}(x, \lambda) \equiv V(x, \lambda)
$$

where $V(x, \lambda)$ is the value function defined by (4.6). Moreover, the optimal control policy is

$$
k^{*}(\lambda)=\left[\frac{b y+\mu-r+\left(\tilde{\sigma}^{2}+\rho \sigma \tilde{\sigma} y\right) \tilde{Z}_{\lambda}(\lambda)}{(1-\gamma) q(y)}\right]^{+}, \quad c^{*}(\lambda)=e^{\frac{\tilde{z}(\lambda)}{\gamma-1}}
$$


Proof. Since $\tilde{Z}$ is a classical solution of (4.18), by definition, it is easy to verify that $\tilde{V}(x, \lambda)$ is a classical solution of (4.7). For any admissible control $\left(k_{t}, c_{t}\right) \in \Pi$, by applying Ito's rule, we can get that

$$
d\left[e^{-\delta t} \tilde{V}\left(X_{t}, \lambda_{t}\right)\right]=-\delta e^{-\delta t} \tilde{V}\left(X_{t}, \lambda_{t}\right) d t+e^{-\delta t} d \tilde{V}\left(X_{t}, \lambda_{t}\right) .
$$

Since $\tilde{V}$ is a solution of (4.7), we can get

$$
\tilde{V}(x, \lambda) \geq \int_{0}^{T} e^{-\delta t} \frac{1}{\gamma}\left(c_{t} X_{t}\right)^{\gamma} d t+e^{-\delta T} \tilde{V}\left(X_{T}, \lambda_{T}\right)+m_{T}+\tilde{m}_{T}
$$

where

$$
\begin{aligned}
& m_{T}=\int_{0}^{T} \sigma y_{t} k_{t} X_{t} \tilde{V}_{x}\left(X_{t}, \lambda_{t}\right) d w_{t} \\
& \tilde{m}_{T}=\int_{0}^{T} \tilde{\sigma}\left(k_{t} X_{t} \tilde{V}_{x}\left(X_{t}, \lambda_{t}\right)+\tilde{V}_{\lambda}\left(X_{t}, \lambda_{t}\right)\right) d \tilde{w}_{t} .
\end{aligned}
$$

Since $k_{t}, y_{t} k_{t}$ and $c_{t}$ are all bounded, by virtue of (4.4), it is not hard to show that

$$
E\left[X_{t}^{m}\right]<\infty, \quad \forall m>0 .
$$

Given this and the fact that $\tilde{Z}, \tilde{Z}_{\lambda}$ are bounded, by virtue of (4.45), (4.49), (4.50), we can get that $m_{T}, \tilde{m}_{T}$ are martingales. Therefore, by taking expectation to (4.48), we have

$$
\tilde{V}(x, \lambda) \geq E \int_{0}^{T} e^{-\delta t} \frac{1}{\gamma}\left(c_{t} X_{t}\right)^{\gamma} d t+E e^{-\delta T} \tilde{V}\left(X_{T}, \lambda_{T}\right) .
$$

If

$$
E \int_{0}^{\infty} e^{-\delta t} \frac{1}{\gamma}\left(c_{t} X_{t}\right)^{\gamma} d t=-\infty
$$

then we must have

$$
\tilde{V}(x, \lambda) \geq E \int_{0}^{\infty} e^{-\delta t} \frac{1}{\gamma}\left(c_{t} X_{t}\right)^{\gamma} d t
$$

If

$$
E \int_{0}^{\infty} e^{-\delta t} \frac{1}{\gamma}\left(c_{t} X_{t}\right)^{\gamma} d t>-\infty
$$

noting that $\gamma<0$, we have

$$
\limsup _{T \rightarrow \infty} E e^{-\delta T} \frac{1}{\gamma}\left(c_{T} X_{T}\right)^{\gamma}=0 .
$$

By virtue of (4.44), we can get

$$
\limsup _{T \rightarrow \infty} E e^{-\delta T} \frac{1}{\gamma} X_{T}^{\gamma}=0 .
$$

Combined with (4.45), (4.43), this implies

$$
\limsup _{T \rightarrow \infty} E e^{-\delta T} \tilde{V}\left(X_{T}, \lambda_{T}\right)=0 .
$$

Taking limsup as $T \rightarrow \infty$ in (4.52), we can get

$$
\tilde{V}(x, \lambda)=E \int_{0}^{\infty} e^{-\delta t} \frac{1}{\gamma}\left(c_{t} X_{t}\right)^{\gamma} d t .
$$


By the definition of $V(X, \lambda)$, we have

$$
\tilde{V}(x, \lambda) \geq V(x, \lambda) .
$$

On the other hand, for $k^{*}, c^{*}$ defined by (4.47), by virtue of (4.43), it not hard to verify that $\left(k^{*}, c^{*}\right) \in \Pi$. Under this control policy, instead of (4.52), now we have an equality

$$
\tilde{V}(x, \lambda)=E \int_{0}^{T} e^{-\delta t} \frac{1}{\gamma}\left(c_{t}^{*} X_{t}\right)^{\gamma} d t+E e^{-\delta T} \tilde{V}\left(X_{T}, \lambda_{T}\right) .
$$

Since $\gamma<0$, we have $\tilde{V}<0$. Thus,

$$
\tilde{V}(x, \lambda) \leq E \int_{0}^{T} e^{-\delta t} \frac{1}{\gamma}\left(c_{t}^{*} X_{t}\right)^{\gamma} d t .
$$

Let $T \rightarrow \infty$. Then we get

$$
\tilde{V}(x, \lambda) \leq E \int_{0}^{\infty} e^{-\delta t} \frac{1}{\gamma}\left(c_{t}^{*} X_{t}\right)^{\gamma} d t .
$$

By the definition of $V$, we must have

$$
\tilde{V}(x, \lambda) \leq V(x, \lambda),
$$

which implies (4.46), combined with (4.55).

REMARK 4.1. For logarithmic utility $U(C)=\log (C),-c x V_{x}+\log (c x)$ is maximum when $c=c^{*}=\left(x V_{x}\right)^{-1}$. The value function had the form

$$
V(x, \lambda)=A \log x+W(\lambda) .
$$

By substituting in the dynamic programming equation (4.7), with log utility, we obtain $A=\delta^{-1}$ and $c^{*}=\delta$ as stated in (3.8).

5. Extensions of the model and future work. In this section, we mention several possible extensions and variants of the models considered in Sections 2-4. These are topics for further research.

A. External income. Suppose that the economic unit receives income from some external source at a positive rate $S_{t}$. The balance equation (2.4) then becomes

$$
d L_{t}=\left[r_{t} L_{t}+I_{t}+C_{t}-Y_{t}-S_{t}\right] d t .
$$

If $S_{t}=S$ is constant and the other assumptions made in Section 2 hold, then the problem of maximizing (2.10) can again be treated by dynamic programming. The dynamic programming equation (4.7) for the value function $V(x, \lambda)$ has an additional term $S V_{x}$. With this additional term, $V(x, \lambda)$ is no longer a homogeneous function of $x$ and there is no "reduced" dynamic programming equation similar to (4.9).

As a special case, one can consider an economic unit with a fixed number of units of capital and with external income only. In this case, $N_{t}=N, I_{t}=0$ and $Y_{t}=0(b=$ $\sigma=0)$. This could be used to model consumer debt, financed by home equity loans. $S_{t}$ represents the consumer's rate of income, financed from salary or other sources. 
B. Price dependent $\mu(P)$. In the model (2.7) for capital unit price fluctuations and also in the log utility case (Section 3), we assumed a "drift term" $P_{t} \mu\left(P_{t}\right) d t$, with $\mu(P)$ depending on price $P$. However, to avoid various technical difficulties, we took $\mu$ constant in Section 4. In that case, the solution $Z(\lambda)$, with $\lambda=\log P$, to the reduced dynamic programming equation (4.11) is bounded together with its derivation $Z_{\lambda}(\lambda)$. One could also consider variable $\mu(P)$, with suitable assumptions which imply that $P_{t}$ is ergodic (or perhaps that the fluctuations of $P_{t}$ about a deterministic trend are ergodic, as in [F-Sh1], [P-R]). Example 3.3 is a special case. In (4.11) and (4.12), $\mu$ and $\tilde{\mu}=\mu-\frac{1}{2} \tilde{\sigma}^{2}$ now depend on $P$, or equivalently on $\lambda$.

C. Parameters dependent on economic factors. It may be unrealistic to assume that the various model parameters remain constant over time. This is especially true if the discount rate $\delta$ is assumed small, and hence the planning horizon is (effectively) long. Several authors have considered modifications of the Merton model, in which interest rates as well as mean rates of return and volatility coefficients depend on economic factors which vary in a random, Markovian way. See [B-P], [F-HH], F-Sh2], [F-P-S], [Z]. A similar extension of the model in the present paper could be considered. For example, one could let the interest rate $r_{t}=r\left(z_{t}\right)$, where $z_{t}$ is some ergodic Markov process with stationary transition probabilities. The value function $V(x, \lambda, z)$ then depends also on the initial state $z_{0}=z$ of the factor process $z_{t}$. The case of a Merton-type model with consumption, and with $r_{t}=z_{t}, Y_{t}=0$, was considered in [F-P] P1 P2].

D. Bounds on rates of investment. In Section 2 no bounds were imposed on the investment rate $I_{t}$. This assumption is appropriate for a small investor portfolio optimization interpretation of the model, in which $Y_{t}=b_{t} N_{t}$ represents a dividend rate. However, it would be less realistic if $K_{t}$ and $L_{t}$ are interpreted as the capital and debt of a productive economic unit, such as a business enterprise. Alternatively, one might impose constraints of the form

$$
-a_{1} K_{t} \leq I_{t} \leq a_{2} K_{t}, \quad a_{1} \geq 0, a_{2} \geq 0 .
$$

\section{REFERENCES}

[B-P] Bielecki, T. R. and Pliska, S. R. (1999), Risk-sensitive dynamic asset management. Appl. Math. Optim. 39, 337-360. MR1675114 (2000d:91062)

[F] Fleming, W. H. (1995), Optimal investment models and risk-sensitive stochastic control. IMA Vol. Math. Appl., 65, 35-45, Springer, New York.

[F-HH] Fleming, W. H. and Hernandez-Hernandez, D.(2003), An optimal consumption model with stochastic volatility, Finance Stoch., Vol. 7, 245-262. MR.1968948 (2004a:91070)

[F-P] Fleming, W. H. and Pang, T. (2004), An Application of Stochastic Control Theory to Financial Economics, SIAM J. of Control and Optimization, Vol. 43, 502-531. MR2086171

[F-R] Fleming, W. H. and Rishel, R. W. (1975), Deterministic and Stochastic Optimal Control. Springer, New York. MR0454768 (56:13016)

[F-Sh1] Fleming, W. H. and Sheu, S. J. (1999), Optimal long term growth rate of expected utility of wealth. Ann. Appl. Probab., Vol. 9, No. 3, 871-903. MR1722286|(2000h:91053)

[F-Sh2] Fleming, W. H. and Sheu, S. J. (2000), Risk-sensitive control and an optimal investment model. Math. Finance, Vol. 10, No. 2, 197-213. MR1802598 (2001k:93137)

[F-So] Fleming, W. H. and Soner, H. M. (1992), Controlled Markov Processes and Viscosity Solutions. Springer, New York. 
STOCHASTIC CONTROL MODEL OF INVESTMENT, PRODUCTION AND CONSUMPTION

[F-St1] Fleming, W. H. and Stein, J. L. (2001), Stochastic inter-temporal optimization in discrete time, in Negishi, Takashi, Rama Ramachandran and Kazuo Mino (ed) Economic Theory, Dynamics and Markets: Essays in Honor of Ryuzo Sato, Kluwer.

[F-St2] Fleming, W. H. and Stein, J. L. (2004), Stochastic optimal control in international finance and debt, J. Banking and Finance, Vol. 28, 979-996.

[F-P-S] Fouque, J. P., Papanicolaou, G. and Sircar, R. (2000): Derivatives in Financial Markets with Stochastic Volatility. Cambridge University Press. MR1768877|(2002g:91082)

[P1] Pang, T. (2002), Stochastic Control Theory and Its Applications to Financial Economics, Ph.D. thesis, Brown University, Providence, RI.

[P2] Pang, T. (2004), Portfolio optimization models on infinite time horizon, Journal of Optimization Theory and Applications, Vol. 22, 119-143. MR2094486

[P-R] Platen, E. and Rebolledo, R. (1996), Principles for modelling financial markets. J. Appl. Probab. 33, 601-613. MF.1401458 (97b:90026)

[Z] Zariphopoulou, T. (2001), A solution approach to valuation with unhedgeable risks. Finance Stoch., Vol. 5(1), 61-82. MR.1807876 (2001j:91086) 